\title{
OXIDATIVE STRESS: ITS ROLE IN AIR POLLUTION AND ADVERSE HEALTH EFFECTS
}

ncreasing concern exists over the adverse effects of air pollution on human health. Epidemiological studies have shown a clear association between cardiovascular morbidity, decreased lung function, increased hospital admissions, mortality, and airborne concentrations of photochemical and particulate pollutants. Human exposure chamber studies of specific pollutants have shown that short term exposure leads to an acute inflammatory effect on normal human airways in a small (10-20\%) proportion of healthy individuals. The consequences of long term exposure to air pollution are more difficult to access but are generally believed to be much worse. Studies in both children and adults have shown that exposure to particulates, nitrogen dioxide and sulphur dioxide, are associated with symptoms of bronchitis. Moreover, exposure to particulates has been related to reduced lung function growth in children and is reversed if the family relocates to a area with lower particulate concentrations.

As with any toxic challenge the obvious solution is to remove, or at least decrease to an acceptable level, the source of trouble. In many countries, air pollution levels have fallen in recent years, while additional measures are in place in several more to decrease concentrations further. It is unlikely however, that these practical measures will completely eliminate the problem, even in the medium term. As a consequence, it has been recognised for some time that there is also a need to improve our understanding of the impact of air pollution on biological systems. For example, a better appreciation of the mechanisms underlying air pollution induced health problems would allow a more targeted approach to remove the most toxic components of air pollution, and could possibly provide a means to decrease individual sensitivity to air pollution. As a consequence of recent research undertaken in a number of different countries, using a range of different approaches, oxidative stress has been identified as a unifying feature underlying the toxic actions of the air pollutants that are causing concern. Oxidative stress, resulting from either increased exposure to oxidants or the presence of decreased antioxidant defences, seems to trigger a number of redox sensitive signalling pathways. There is now a strong body of evidence to indicate that the pulmonary inflammatory response that arises following exposure to a pollution episode, is mediated via oxidant signalling pathways. Moreover, it appears likely that an individual's sensitivity to pollution is related, in part, to their pulmonary antioxidant defences.

\section{WHAT IS OXIDATIVE STRESS?}

Correspondence to: Professor Frank J Kelly, Environmental Research Group, School of Health \& Life Science, Franklin-Wilkins Building, King's College, London, 150 Stamford Street, London SE 1 9NN, UK; frank.kelly@kcl.ac.uk
Oxidative stress is a relatively new term in biology that was first introduced by Sies in $1991 .{ }^{1}$ In this book, oxidative stress was defined as "a disturbance in the prooxidant-antioxidant balance in favour of the former, leading to potential damage". Since then many other definitions have been proposed, all of which attempt to explain a process which essentially involves the flow of electrons from one molecule to another within a biological setting. The importance of this process lies in the reactivity of the molecules involved. Under normal circumstances, electrons orbit around atoms in pairs, having opposite spins. When an atom has a single unpaired electron, its reactivity increases markedly, and it is referred to as a free radical. In a biological setting, free radicals are potentially very dangerous since they can react indiscriminately with neighbouring molecules. This process of "electron stealing" leads to oxidation, and often inactivation of target molecules. If these reactions are numerous they can cause extensive cellular damage. The extent of damage is related to the availability of neutralising antioxidant defences, as these specialised molecules preferentially react with free radicals, while the products of these reactions often have low toxicity. The damage arising from aberrant free radical activity is often loosely referred to as oxidative stress. In its simplest form then, oxidative stress is a potentially harmful process, that occurs when there is an excess of free radicals, a decrease in antioxidant defences, or a combination of these events (fig 1). To complicate matters, it has recently been shown that low levels of oxidative stress are involved in signalling pathways within the cell, especially those involved in regulating immune responses. ${ }^{2}$ Hence not all forms of oxidative stress are damaging to the cell, and indeed some may be very beneficial.

Oxidative stress can arise for many reasons, including consumption of alcohol, medications, trauma, cold, air pollutants, toxins, and radiation. The scope of this review is limited to the role 
Oxidative stress

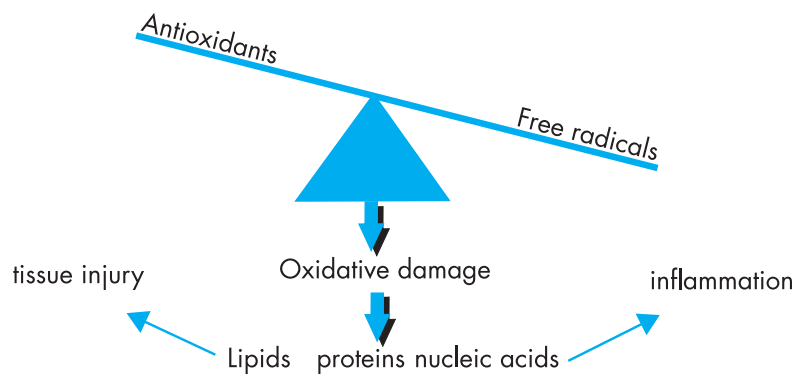

Figure 1 Oxidative stress exists when there is an excess of free radicals over antioxidant defences. As a consequence, free radicals attack and oxidise other cell components such as lipids (particularly polyunsaturated lipids), proteins, and nucleic acids. This leads to tissue injury and in some cases, the influx of inflammatory cells to the sites of injury.

oxidative stress plays in air pollution induced health effects and in particular, those events that occur at the surface of the lung.

\section{AIR POLLUTION AND OXIDATIVE STRESS}

Ambient air contains a range of pollutants, the exact combination of which varies from one microenvironment to the next. Many of the individual pollutants that make up this ambient mix are free radicals (for example, nitrogen dioxide) or have the ability to drive free radical reactions (for example, ozone and particulates). As a consequence, exposure to a wide range of air pollutants gives rise to oxidative stress within the lung, and this appears to initiate responses that are particularly dangerous to susceptible members of the population. One of these responses is the influx of inflammatory cells to the lung. This highly orchestrated series of events can lead to a second wave of oxidative stress in the lung, since activated inflammatory cells also generate and release large quantities of free radicals. In the absence of any invading organisms to kill, these free radicals attack local tissue components and cause cell injury. Prior to considering these reactions in more detail, it is worth reviewing the impact and chemistry of the three major pollutants of concern.

\section{Ozone}

Ozone is a highly reactive gas and a major constituent of photochemical smog. Breathing only slightly increased concentrations of ozone (60-120 ppb) results in a range of respiratory symptoms in a small proportion (10-20\%) of the healthy population. ${ }^{3}$ Symptoms include decreased lung function, increased airway hyperreactivity, and pulmonary inflammation. Those individuals with pre-existing conditions such as asthma and chronic obstructive pulmonary disease generally experience an exacerbation of their symptoms.

Ozone is a relatively insoluble gas. It is very reactive, and uptake is directly related to reactions with substrates present in the lung lining fluid, the first compartment it encounters on entering the lung. Langford et al referred to this mechanism as "reactive absorption". ${ }^{4}$ The uptake of ozone is thus related not only to its concentration but also availability of substrates within the lung lining fluid compartment. Following reaction with a target substrate, ozone is consumed, disabling it from transiting the lung lining fluid compartment. Cellular responses to ozone are therefore not the result of direct reaction of ozone with cell surface components, but are mediated through a cascade of secondary, free radical derived, ozonation products.

\section{Nitrogen dioxide}

The major source of nitrogen dioxide in urban areas is traffic, with ambient concentrations fluctuating with the morning and evening rush hours. Indoors, cooking with gas appliances and cigarette smoke are the major sources. Animal studies have shown that high concentrations of nitrogen dioxide can damage the lung, while cell culture experiments indicate that exposure increases cell permeability and injury. In a series of studies using a combination of environmental chamber exposure and fibreoptic bronchoscopy with bronchoalveolar lavage, Sandström and colleagues ${ }^{5}$ have shown that both single and multiple exposures of humans to occupational concentrations of nitrogen dioxide result in a time dependent inflammatory response in the lung.

Nitrogen dioxide is a nitrogen centred free radical with limited solubility in aqueous solutions. Like ozone, it reacts with substrates present in the lung lining fluid compartment, and is therefore unlikely to interact directly with the pulmonary epithelium. ${ }^{6}$ Instead, it is the oxidised species arising from a reaction between nitrogen dioxide and the lung lining fluid compartment, that is responsible for initiating the signalling cascade which brings the inflammatory cells into the lung.

\section{Particulates}

Epidemiological studies have consistently reported associations between particulate air pollution, especially particles less than $10 \mu \mathrm{m}\left(\mathrm{PM}_{10}\right)$ in aerodynamic diameter, and adverse health effects, increasing morbidity and mortality. ${ }^{7}$ It is by no means clear how exposure to PM, typically as low as $30 \mu \mathrm{g} / \mathrm{m}^{3}$, can produce these health effects and which components of PM mediate them. Although epidemiological evidence suggests that it is the fine $\left(\mathrm{PM}_{2.5}\right)$ or ultrafine $\left(\mathrm{PM}_{0.1}\right)$ fraction that contains the toxic components, there is no general agreement. The wide spectrum of disease endpoints (from cardiovascular death to asthma attack) suggests that more than one component may be driving the health effects.

Ambient particles contain a large number of soluble metals including transition metals that are capable of redox cycling. The idea has therefore developed that oxidative stress underlies much of the toxicity of ambient particles. ${ }^{8}$ In the USA, studies involving residual oil fly ash (ROFA) have helped develop and refine this theory. ROFA contains about $10 \%$ by weight of water soluble $\mathrm{Fe}, \mathrm{Ni}$, and $\mathrm{V}$, and its intratracheal instillation in rats leads to aldehyde generation. Moreover, oxidative stress has been shown by electron spin resonance (ESR) measurements in animals instilled with ROFA, ${ }^{9}$ and exposure to aqueous extract of ROFA produces effects similar to those of unfractionated ROFA. Pulmonary inflammation induced by ROFA is reproducible by installation of mixtures of $\mathrm{V}, \mathrm{Ni}$, and $\mathrm{Fe}$ in proportions similar to those seen in the water soluble fraction of ROFA. Likewise, exposure of phagocytic cells to ambient particles collected from different urban settings causes oxidative stress which correlates with the iron content of the particles. This and other work has led to the suggestion that the dose of bioavailable transition metal, rather than particulate mass, may be the primary determinant of the acute inflammatory response. ${ }^{10}$

Evidence is also accumulating to suggest that organic components carried on the particle surface play an important role in mediating the toxic effect. For example, polycyclic aromatic hydrocarbons (PAHs) can induce oxidative stress indirectly, through biotransformation by cytochrome $\mathrm{P} 450$ and dihydrodiol dehyrrogenase to generate redox active quinones that act as catalysts for free radical production. Other work has highlighted the potential role of particle bound endotoxin in driving the toxicity associated with particulate exposure. 
Table 1 Lung lining fluid antioxidant defences

\begin{tabular}{ll}
\hline Type & Name \\
\hline Low molecular weight & Glutathione (GSH) \\
& Tocopherol (vitamin E) \\
& Ascorbic acid \\
& Uric acid \\
& $\alpha$ tocopherol \\
& Glutathione peroxidase \\
Enzymatic & Superoxide dismutase \\
& Catalase \\
Metal binding & Caeroplasmin \\
& Transferrin \\
\hline
\end{tabular}

\section{LUNG LINING FLUID ANTIOXIDANT DEFENCES}

The lung, owing to its function and large surface area, is clearly a primary target organ for oxidative injury. It is therefore logical that the lung has a robust extracellular antioxidant defence system to protect against undue oxidation of its delicate pulmonary epithelial cells. Oxidative damage to the blood/gas barrier would play havoc with the gaseous exchange processes that are essential for normal life. Indeed, it is likely that this type of injury underlies respiratory failure in diseases such as emphysema and cystic fibrosis. ${ }^{11}$

As mentioned above, when a pollutant first enters the lung, the first interface it encounters is the lung lining fluid. This compartment consists of secretions from underlying lung and resident immune cells, as well as plasma derived exudate. Lung lining fluid can be sampled by bronchoalveolar lavage, and the diluted form of lung lining fluid obtained can be analysed by a range of techniques such as high pressure liquid chromatography (fig 2). Using this approach, it has been established that lung lining fluid contains a similar range of low molecular weight antioxidants to blood plasma, including reduced glutathione, ascorbic acid (vitamin C), uric acid, and $\alpha$ tocopherol (vitamin E). There are marked differences in the concentration of these antioxidant defences between the upper and lower respiratory tract. Lung lining fluid obtained from the lower respiratory tract contains abundant amounts of reduced glutathione and ascorbate, but low concentrations of uric acid and $\alpha$ tocopherol. ${ }^{12}$ In contrast, lung lining fluid from the nasal cavity contains uric acid in large quantities, with much smaller amounts of reduced glutathione (GSH) and vitamin C. In addition to these low molecular weight antioxidants, lung lining fluid contains antioxidant enzymes such as superoxide dismutase and catalase, as well as the metal binding proteins caeruloplasmin and transferrin (table 1).

\section{POLLUTANT-ANTIOXIDANT INTERACTIONS IN LUNG LINING FLUID}

Studies employing ${ }^{18} \mathrm{O}$ labelled ozone have revealed that lung lining fluid takes up proportionally more ozone than either the lung lining fluid cell fraction or lung tissue. ${ }^{13}$ These data, combined with a range of in vitro studies showing the interaction between antioxidants and a range of pollutants, ${ }^{714}$ have led to the general belief that antioxidants present in lung lining fluid protect the lung from oxidative challenge arising from air pollution. When ozone reacts with non-antioxidant substrates in lung lining fluid, such as protein or lipid, secondary oxidation products arise; it is possible that these transmit toxic signals to the underlying pulmonary epithelium. Similarly, exposure to

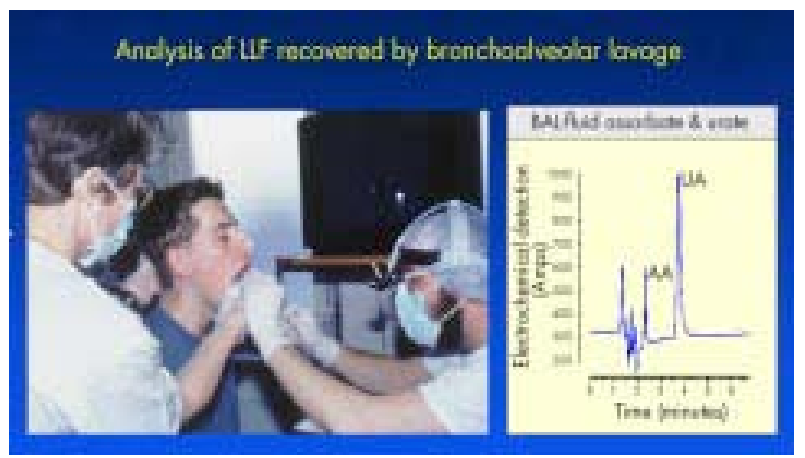

Figure 2 Subject being prepared for fibreoptic bronchoscopy to obtain bronchoalveolar lavage (left panel). Analysis of ascorbic acid (AA) and uric acid (UA) concentration in lavage fluid by high pressure liquid chromatography (right panel).

nitrogen dioxide of sufficient concentrations to overcome the endogenous antioxidant defences, will result in the generation of secondary oxidant species that lead to the same effects.

The mechanisms underlying ambient particulate toxicity are likely to be somewhat more complicated. Oxidising species such as transition metals and certain organic compounds located on the surface of particles, will interact with, and deplete lung lining fluid antioxidants in the same manner as gaseous pollutants. As a consequence, these particles will arrive at the lung surface in a less active form (fig 3). However, surface reactivity is not the only aspect of particle toxicity. A number of groups have produced evidence, which indicates that particle size is an important determinant of reactivity. On a mass basis, ultrafine particles have a much greater surface area and therefore capacity to carry toxic components into the deep lung. However, Brown and colleagues ${ }^{15}$ have shown that even ultrafine particles without transition metals on their surface induce marked inflammation in rat lungs, indicating that particle size itself may be a critical factor.

The rules that govern the balance between beneficial and detrimental interactions in the lung lining fluid compartment are not well established, but these may contribute in part to the sensitivity of individuals to air pollution. ${ }^{16}$ The most simplistic viewpoint would be that the greater the range and concentration of antioxidant defences on the lung surface, the better the level of protection from oxidant air pollutants. If this were the case however, subgroups of the population recognised to be susceptible to air pollution should have decreased lung lining fluid antioxidant defences. This has indeed been found in asthmatics-a susceptible subgroup in that they have markedly decreased concentrations of ascorbic acid (vitamin C) in lung lining fluid compared to healthy control subjects. ${ }^{17}$ However, the same was not found to be true of those healthy subjects who happen to show the largest decrease in lung function following ozone challenge. ${ }^{18}$ of course it could be argued that a decrease in lung function is not necessarily a bad response when faced with an ozone episode. At this time however, much more work is needed to validate the assumption that lung lining fluid antioxidants perform a critical role in reducing the toxic consequences of oxidant air pollutants.

\section{OXIDANT EVENTS AT THE SURFACE OF THE LUNG}

On reaching the lung surface, secondary oxidation products arising from oxidant pollutants initiate a number of cellular responses. These include cytokine generation, adhesion molecule expression, and tight junction modification. These 


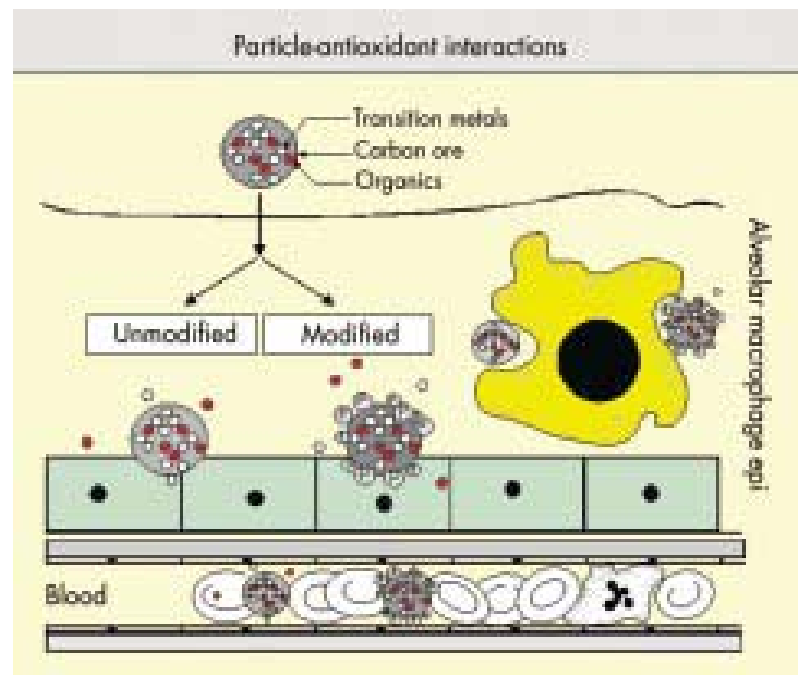

Figure 3 Ambient particles contain a range of transition metals and organics on the surface of the carbon core. These are capable of undergoing redox chemistry with the antioxidant defences within the lung lining fluid. Although this will lead to the consumption of the protective antioxidant defences it will also result in a less active particle reaching the surface of the lung.

responses lead to the influx of inflammatory cells to the lung in the absence of a pathogenic challenge; lung permeability is increased and oedema develops. The nature and extent of these responses are variable, and often not related within an individual. Recently, Pryor in collaboration with Utell's group addressed these issues. ${ }^{19}$ They were able to show that lipid oxidation products (aldehydes) are present in lung lining fluid, and that the concentration of one particular aldehyde, namely nonanal, increases following four hours exposure to $0.22 \mathrm{ppm}$ ozone. Although the concentration of another aldehyde, hexanal, also increased post-exposure, the change was not significant. Aldehydes, although rapidly metabolised, are toxic to cells, and as such, it is likely that nonanal and/or other aldehydes generated in the lung lining fluid compartment on exposure to air pollutants, are responsible for at least some of the responses of the underlying lung cells.

\section{PULMONARY INFLAMMATION AND OXIDATIVE STRESS}

A common feature of all forms of air pollution challenge is the influx of activated inflammatory cells to the lung. In this way the generation of free radicals by activated inflammatory cells is also involved in the oxidative stress associated with air pollution. The events that lead to the influx of inflammatory cells to the lung in response to pollution are now becoming clear. Signals, perhaps activated lipids in the case of ozone, lead to the activation of transcription factors such as nuclear factor $\kappa \mathrm{B}(\mathrm{NF} \kappa \mathrm{B})$ and increased expression of a range of proinflammatory chemokines and cytokines. These signalling molecules lead to the upregulatin of a range of adhesion molecules, both on the endothelial cell surface and by the inflammatory cells. The respective adhesion molecules recognise each other, and direct movement of the immune cells from the vasculature into the lung tissue.

Immune cells such as neutrophils, eosinophils, and macrophages possess a membrane bound flavoprotein, cytochrome b245 NADPH oxidase, which is induced during cell activation. Using molecular oxygen, the NADPH oxidase produces superoxide anions. These reactive oxygen species are removed by

\section{Key points}

- A thin layer of fluid richly invested with antioxidant defences covers the surface of the lung.

- Oxidative stress exists when free radical production exceeds available antioxidant defences.

- The major air pollutants causing public health concern, ozone, nitrogen dioxide, and particulates (PM), all give rise to oxidative stress in the lung.

- Healthy subjects have a range of antioxidant defences in lung lining fluid, but asthmatics - a group sensitive to air pollution-have lower levels, particularly of vitamin C.

superoxide dismutase, which is present at the epithelial surface of the lung. ${ }^{20}$ Removal in this way results in the generation of hydrogen peroxide, which, because of its relatively low reactivity, can easily pass across cell membranes, where it may activate intracellular signalling pathways, or lead to the generation of other reactive oxygen species. For example, in the presence of transition metals, hydrogen peroxide leads to the production of the more toxic, hydroxyl radical $(\mathrm{OH})$. Alternatively, myleoperoxidase, which is also released by the neutrophil, uses hydrogen peroxide as a substrate, and produces hypochlorous acid ( $\mathrm{HOCl})$, another potent oxidant.

The influx, and subsequent activation, of inflammatory cells such as neutrophils to the lung therefore represents a second wave of oxidative stress. As in the case of the primary oxidant challenge, the endogenous antioxidant defences in the lung lining fluid compartment are probably crucial in dictating the extent of the "response" to this second challenge, which manifests some 6-18 hours after the initial exposure.

\section{WHAT QUESTIONS STILL REMAIN TO BE ANSWERED?}

There is now a substantial body of evidence supporting a role for oxidative stress in mediating the toxicity of ambient pollution. Individual air pollutants oxidatively challenge the lung directly through their free radical activity, and by causing an influx of activated inflammatory cells to the lung. In healthy subjects, the lung appears to be sufficiently endowed with protective antioxidant defences in the lung lining fluid compartment, the first interface for inspired pollutants. Asthmatics, a group sensitive to air pollution, have compromised lung lining fluid antioxidant status. Given these findings it seems plausible to suggest that antioxidant defences on the surface of the lung play an important role in regulating the response of any one individual to an air pollution episode. Second, although nitrogen dioxide is a free radical and ozone a powerful oxidant, the oxidant activity of ambient particles appears to be driven by specific components adsorbed on to their surface such as transitional metals and certain organic compounds. Therefore it may be feasible to decrease the level of these specific surface components through targeted regulation, and thus reduce the toxicity of ambient particulates.

Even though considerable progress has been made in identifying the mechanisms underlying the risks to health of air pollution, considerably more work is required. From an oxidative stress point of view the following questions still need to be addressed.

(1) A better understanding of lung lining fluid antioxidant status in susceptible groups is needed. This research should include an examination of the antioxidant status of this compartment in the very young and elderly. 
(2) An improved understanding of those factors that regulate antioxidant defences on the lung surface is required.

(3) Investigations are needed to determine whether improved antioxidant status, in those with lung lining fluid deficiencies, results in improved protection from oxidant air pollutants.

(4) A better understanding of how the surface chemistry of particles influences their oxidative activity would be useful.

\section{ACKNOWLEDGEMENTS}

I would like to thank Dr Ian Mudway who helped in the preparation of the diagrams for this article. The air pollution studies in the author's laboratory are supported by the MRC in the UK and the EU.

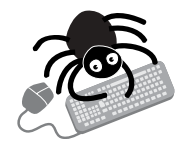

Additional references appear on the Occupational and Environmental Medicine website [www.occenvmed.com]

\section{REFERENCES}

1 Sies H. Oxidative stress II. In: Oxidants and antioxidants. London: Academic Press, 1991.

- This book introduces for the first time the concept of oxidative stress in biological systems. The term is now used extensively in all aspects of disease and is particularly relevant to the lung, for which a number of respiratory diseases involve oxidative stress.

2 Forman HJ, Torres M, Fukuto J. Redox signalling. Mol Cell Biochem 2002;234-235:49-62.

- Free radicals have recently been shown to be involved in a multiplicity of physiological responses through modulation of signalling pathways. This is an excellent review of the current understanding in this area written by a group at the forefront of this work.

3 Mudway IS, Kelly FJ. Ozone and the lung: a sensitivity issue. Mol Aspects Med 2000;21:1-48

4 Langford SD, Bidani A, Postlethwait EM. Ozone-reactive absorption by pulmonary epithelial lining fuid constituents. Toxicol Appl Pharmacol $1995 \cdot 132: 122-30$.

5 Sandström T, Stjernberg N, Eklund A, et al. Inflammatory cell response in bronchoalveolar lavage fluid after nitrogen dioxide exposure of healthy subjects: a dose-response study. Eur Respir J 1991;4:332-9.

6 Kelly FJ, Tetley T. Nitrogen dioxide depletes uric acid and ascorbic acid but not glutathione from lung lining fluid. Biochem J 1997;325:95-9.

7 Samet JM, Dominici F, Curriero FC, et al. Fine particulate air pollution and mortality in 20 U.S. cities, 1987-1994. N Engl J Med 2000;343:1742-9.

- This epidemiological study confirms, and builds on, earlier studies in the US indicating that there is consistent evidence that the levels of fine particulate matter in the air are associated with the risk of death from all causes and from cardiovascular and respiratory illnesses.

8 Li XY, Gilmour PS, Donaldson K, et al. Free radical activity and pro-inflammatory effects of particulate air pollution (PM10) in vivo and in vitro. Thorax 1996;51:1216-22.

- This was one of the first descriptions of free radical activity of ambient PM.

9 Kadiiska MB, Mason RP, Dreher KL, et al. In vivo evidence of free radical formation in the rat lung after exposure to an emission source air pollution particle. Chem Res Toxicol 1997; 10:1 104-8.

- This is one of the first studies to show in an in vivo setting free radical activity associated with ambient particulates.

10 Costa DL, Dreher KL. Bioavailable transition metals in particulate matter mediate cardiopulmonary injury in health and compromised animal models. Environ Health Perspect 1997; 105(suppl 5):1053-60.

11 Brown RK, Kelly FJ. The role of free radicals in the pathogenesis of cystic fibrosis. Thorax 1994;49:738-42.

- This paper overviews the imbalance in antioxidants and oxidants in cystic fibrosis and discusses how the ensuing oxidative stress may. contribute to the long term decline in lung function in cystic fibrosis patients.

12 Kelly FJ, Buhl R, Sandström T. Measurement of antioxidants, oxidants and oxidant products in bronchoalveolar lavage fluid. Eur Respir Rev 1999;9:93-8.

13 Hatch GE, Slade R, Harris LP, et al. Ozone dose and effect in humans and rats. A comparison using oxygen-1 8 labeling and bronchoalveolar lavage. Am J Respir Crit Care Med 1994;150:676-83.

- In this study the authors elegantly showed that ozone preferentially reacts with molecular components of lung lining fluid rather than resident cells or lung tissue.

14 Mudway IS, Kelly FJ. Modelling the interactions of ozone with pulmonary epithelial lining fluid antioxidants. Toxicol Appl Pharmacol 1998;48:91-100

15 Brown DM, Stone V, Findlay $P$, et al. Increased inflammation and intracellular calcium caused by ultrafine carbon black is independent of transition metals or other soluble components. Occup Environ Med 2000:57:685-91.

16 Kelly FJ, Mudway IS, Krishna MT, et al. The free radical basis of air pollution: focus on ozone. Respir Med 1995;89:647-56.

17 Kelly FJ, Mudway I, Blomberg A, et al. Altered lung antioxidant status in patients with mild asthma. Lancet 1999:354:482-3.

- This paper examines the antioxidant defences on the surface of the lung in asthmatics and compares these with those in age and gender matched healthy control subjects. It reports that the concentration of the protective antioxidant, ascorbic acid (vitamin C) is drastically reduced in lung lining fluid, while the concentration of oxidised glutathione (a sign of oxidative stress) is increased in asthmatics.

18 Mudway IS, Stenfors N, Blomberg A, et al. Differences in basal airway antioxidant concentrations are not predictive of individual responsiveness to ozone: a comparison of healthy and mild asthmatic subjects. Free Radic Biol Med 2001;31:962-74.

19 Frampton MW, Pryor WA, Cueto R, et al. Ozone exposure increases aldehydes in epithelial lining fluid in human lung. Am J Respir Crit Care Med 1999;159:1134-7.

20 Oury TD, Chang LY, Marklund SL, et al. Immunocytochemical localization of extracellular superoxide dismutase in human lung. Lab Invest 1994;70:889-98. 